\title{
An ensemble framework for assessing solutions of interval programming problems
}

DOI:

10.1016/j.ins.2018.01.006

\section{Document Version}

Accepted author manuscript

Link to publication record in Manchester Research Explorer

\section{Citation for published version (APA):}

Sun, J., Gong, D., Zeng, X., \& Geng, N. (2018). An ensemble framework for assessing solutions of interval programming problems. Information Sciences, 436-437, 146-161. https://doi.org/10.1016/j.ins.2018.01.006

\section{Published in:}

Information Sciences

\section{Citing this paper}

Please note that where the full-text provided on Manchester Research Explorer is the Author Accepted Manuscript or Proof version this may differ from the final Published version. If citing, it is advised that you check and use the publisher's definitive version.

\section{General rights}

Copyright and moral rights for the publications made accessible in the Research Explorer are retained by the authors and/or other copyright owners and it is a condition of accessing publications that users recognise and abide by the legal requirements associated with these rights.

\section{Takedown policy}

If you believe that this document breaches copyright please refer to the University of Manchester's Takedown Procedures [http://man.ac.uk/04Y6Bo] or contact uml.scholarlycommunications@manchester.ac.uk providing relevant details, so we can investigate your claim.

\section{OPEN ACCESS}




\title{
An Ensemble Framework for Assessing Solutions of Interval Programming Problems
}

\author{
Jing $\operatorname{Sun}^{a}$, Dunwei Gong ${ }^{b, c *}$, Xiaojun Zeng ${ }^{d}$, Na Geng ${ }^{e}$ \\ ${ }^{a}$ College of Sciences, Huaihai Institute of Technology, Lianyungang, 222005, China \\ ${ }^{b}$ School of Information and Control Engineering,China University of Mining and \\ Technology, Xuzhou, 221116, China \\ ${ }^{c}$ School of Information Science and Technology, Qingdao University of Science and \\ Technology, Qingdao, 266061, China \\ ${ }^{d}$ School of Computer Science, The University of Manchester, Manchester, M13 9PL, UK \\ ${ }^{e}$ School of Electrical engineering and automation, Jiangsu Normal University, Xuzhou, \\ 221116, China
}

\begin{abstract}
Interval programming is a commonly used technique in real-world situations. Its related theories and methods have been widely researched. There are a variety of approaches for assessing solutions of an interval programming problem due to the particularity of intervals. It is well-known that different assessing approaches may produce different optimal solution(s) for the same interval programming problem, and it is rather difficult to choose from these assessing approaches for users, especially for those who have little knowledge about interval arithmetic, which greatly restricts its extensive applications.

In this paper, we develop an ensemble framework for assessing solutions of interval programming problems. At the start, interval dominance rules are defined, and their correlations are described via exclusion, inclusion and equivalence; then, a rule reduction strategy is developed through inspecting the impact of different rules on the sorting of solutions, and a novel ensemble dominance relation for interval programming is proposed to evaluate solutions; furthermore, their complexities are analyzed; finally, the experimental results empirically validate the correctness and effectiveness of the proposed framework.
\end{abstract}

\footnotetext{
${ }^{*}$ Corresponding author. Tel.:+86 51683995312.

Email address: dwgong@vip.163.com (Dunwei Gong ${ }^{b, c *}$ )
} 
Keywords: Uncertain programming, interval programming, numerical optimization, evolutionary algorithm, ensemble dominance

\section{Introduction}

Mathematical programming is an important discipline in modern mathematics, and its theories and methods have permeated into a large number of research fields, such as social science [41], management science [31] and engineering technology [3]. In a conventional mathematical programming model, its coefficients are usually exact. However, most of the models are inherently uncertain, or although some problems are precise, the uncertainty of cognition due to the scarcity of data, the inaccuracy of measured data, or the simplification of models leads to uncertain coefficients [33, 46].

Interval programming is a branch of mathematical programming for handling uncertainty. In a practical application, compared with the probabilistic distribution of a stochastic variable and the possibilistic distribution of a fuzzy number, the information of an interval, i.e., its midpoint, width, lower and upper bounds, are relatively easy to get [49]. Furthermore as Vladik Kreinovich argued, the most appropriate foundation for representing and processing different types of uncertainty is intervals [25]. As a result, interval programming has attracted extensive attentions and been applied into many different fields, such as profit maximization [29], water resources allocation [16], automobile design [23] and smart grid [28].

During the last three decades, abundant research results about interval programming theories and methods have been obtained [1, 6, 18, 21, 36, 45]. In numerical optimization, the approaches for solving them can be grouped into three categories: satisficing approaches, optimizing approaches and minimax regret approaches. The satisficing approaches transform an interval programming model into one or more deterministic model(s) by using ordering relations or possibility degrees between intervals, and then solutions of the original model are evaluated according to the values of the transformed deterministic objective function(s) $[1,2,6,9,10,21,33]$. The optimizing approaches put forward the concepts of weak or strong optimal solutions for interval linear programming $[19,26,30,36]$ or extend the concept of efficiency used in conventional multi-objective programming to interval environment $[5,17,34]$. While the minimax regret approaches transform an interval programming model into a deterministic one by borrowing the minimax regret criterion from the decision theory [20,37]. 
Evolutionary algorithms (EAs) are stochastic global optimization approaches inspired by nature evolution and genetic mechanisms, and have superior capabilities in handling complicated problems. There exist two classes of methods to solve interval programming problems by EAs: one is transforming them into deterministic models which is similar to the above satisficing approaches, and then employing EAs to tackle the transformed models $[4,14,22,38]$; the other is proposing a novel dominance relation by using an ordering relation or a possibility degree between intervals, and then adopting EAs to directly handle them [12, 13, 27, 39, 47, 48].

Clearly, the pivotal technique of both numerical and evolutionary optimization is assessing solutions with the help of some particular approaches except for optimization algorithms. It is easy to understand that different assessing approaches will derive different interval dominance relations; different dominance relations likely generate different optimal solution sets for the same interval programming problem even though the same algorithm is used. So which solution set is favorable is really a key problem for users. In a word, although interval uncertainty is easily got and expressed, a variety of approaches for assessing solutions are daunting, especially for users who have little knowledge about interval arithmetic, which will largely restrict extensive applications of interval programming. If employing several approaches to assess solutions of an interval programming problem in a self assembling framework, the produced optimal solution(s) will not rely on a single assessing approach. In this way, users will free themselves from choosing an appropriate approach to assess solutions and focus on the optimization algorithms. So a great deal of optimization algorithms can be conveniently used to solve interval programming problems, and it will also boost more applications of interval programming.

Based on the above consideration, we develop an ensemble framework for assessing solutions of interval programming problems in this paper. The correlations between approaches for assessing solutions are first investigated through going into the optimal solution sets of an interval programming model corresponding to them, and then a method for assembling them is put forward. The method includes two modules. One is reducing approaches for assessing solutions, which will be accomplished before optimization and whose role is reducing an approach set by discerning equivalent approaches so as to make diverse decisions during optimization, thus enhancing their ensemble efficiency. The other is integrating approaches, which can be embedded into any optimization algorithm and whose role is comprehensively 
assessing solutions by assembling evaluation results of multiple approaches during optimization. Some preliminary results of this study were presented in [40]. In this paper, we expound the methodology and develop the following new characters:

1) Reviewing the related theories and methods for interval programming.

2) Providing the detailed proof of each proposition.

3) Showing the propositions of ordering relations between intervals, analyzing the impacts of different approaches for assessing solutions on optimal solution sets and revealing the proposed ensemble dominance via examples.

4) Adding the detailed descriptions of two other approaches for assessing solutions.

5) Adding two bi-objective test models and conducting some new experiments to support the empirical conclusions.

The contributions of this study can be summarized as follows: 1) systematically researching on approaches for assessing solutions of interval programming problems. Three relations, which are equivalence, inclusion and exclusion, are adopted to describe their correlations in detail; in addition, the three relations are equivalently described on the basis of optimal solution(s) of any solution set for an interval programming problem to easily discern the correlation between approaches; 2) presenting a reduction strategy for assessing approaches so that the ensemble result will not be affected by several equivalent approaches and most of users can accept it, thus enhancing the ensemble efficiency; 3) integrating multiple approaches to assess solutions of an interval programming problem to avert the trouble in choosing a suitable approach and produce satisfactory optimal solution(s).

The remainder of this paper is organized as follows. Section 2 formulates interval programming models and reviews the related work. In Section 3, approaches for assessing solutions of an interval programming problem are defined as interval dominance rules for the sake of narrative, and their correlations are elaborated. Section 4 develops a reduction strategy for interval dominance rules, suggests an ensemble dominance relation and theoretically analyzes the complexities. The proposed framework was empirically tested on three groups of experiments in Section 5 to verify its rationality and effectiveness. Section 6 provide the main conclusions of this paper and put forwards some new directions for future research. 


\section{Related work}

\subsection{Interval programming models}

An interval is defined as $a=\left[a^{L}, a^{U}\right]$, where $a^{L}, a^{U} \in R$ and $a^{L} \leq a^{U} ; a^{L}$ and $a^{U}$ are the lower and upper bounds of $a$, respectively. We denote the midpoint and width of $a$ as $m(a)=\frac{a^{L}+a^{U}}{2}$ and $w(a)=a^{U}-a^{L}$, respectively. When $a^{L}=a^{U}, a$ is degraded into a point. We also denote the set of all closed intervals on $R$ as $I(R)$.

Without loss of generality, an interval programming model is formulated as follows.

$$
\begin{aligned}
& \max f(\mathbf{x}, \mathbf{c})=\left(f_{1}\left(\mathbf{x}, \mathbf{c}_{1}\right), f_{2}\left(\mathbf{x}, \mathbf{c}_{2}\right), \cdots, f_{m}\left(\mathbf{x}, \mathbf{c}_{m}\right)\right) \\
& \text { s.t. } g_{j}\left(\mathbf{x}, \mathbf{a}_{j}\right) \leq\left[b_{j}^{L}, b_{j}^{U}\right], j=1,2, \cdots, l, \\
& \quad \mathbf{x} \in \mathbf{S} \subseteq \mathbf{R}^{n}
\end{aligned}
$$

where

$\mathbf{-} \mathbf{x}=\left(x_{1}, x_{2}, \cdots, x_{n}\right)^{T}$ is an $n$-dimensional decision vector;

- $\mathbf{S}$ is a decision space of $\mathbf{x}$;

$-\mathbf{c}_{i}=\left(c_{i 1}, c_{i 2}, \cdots, c_{i k}\right)^{T}, i=1,2, \cdots, m$, and $\mathbf{a}_{j}=\left(a_{j 1}, a_{j 2}, \cdots, a_{j k}\right)^{T}, j=$ $1,2, \cdots, l$, are interval vector coefficients; $c_{i p}=\left[c_{i p}^{L}, c_{i p}^{U}\right]$ and $a_{j p}=\left[a_{j p}^{L}, a_{j p}^{U}\right]$, $p=1,2, \cdots, k$ are the $p$-th components of $\mathbf{c}_{i}$ and $\mathbf{a}_{j}$, respectively; when all $c_{i p}$ and $a_{j p}$ degrade to points, model (1) is a deterministic one;

$-f_{i}\left(\mathbf{x}, \mathbf{c}_{i}\right), i=1,2, \cdots, m$, is the $i$-th objective function with interval coefficients; when $m=1$, model (1) is a single-objective programming model; when $m>1$, model (1) is a multi- objective programming model; each objective value in model (1) is an interval because of its interval coefficients, denoted as $f_{i}\left(\mathbf{x}, \mathbf{c}_{i}\right) \triangleq\left[f_{i}^{L}\left(\mathbf{x}, \mathbf{c}_{i}\right), f_{i}^{U}\left(\mathbf{x}, \mathbf{c}_{i}\right)\right], i=1,2, \cdots, m$. When the objective function is linear, model (1) is an interval linear programming model; when the objective function is non-linear, model (1) is an interval non-linear programming model;

$-g_{j}\left(\mathbf{x}, \mathbf{a}_{j}\right) \leq\left[b_{j}^{L}, b_{j}^{U}\right], j=1,2, \cdots, l$ are constraints of model (1); when $l=0$, Eq. (1) is an unconstraint programming model.

In this paper, we mainly focus on the following unconstraint interval programming model:

$$
\begin{aligned}
& \max f(\mathbf{x}, \mathbf{c})=\left(f_{1}\left(\mathbf{x}, \mathbf{c}_{1}\right), f_{2}\left(\mathbf{x}, \mathbf{c}_{2}\right), \cdots, f_{m}\left(\mathbf{x}, \mathbf{c}_{m}\right)\right) \\
& \text { s.t. } \mathbf{x} \in \mathbf{S} \subseteq \mathbf{R}^{n} .
\end{aligned}
$$


The solutions of model (2) cannot be evaluated by the ordering relation of exact value due to its interval objective values. The comparison of intervals will be elaborated in the following subsection.

\subsection{Ordering relations and possibility degrees of intervals}

The set of ordered pair of binary relation $I(R) \times I(R)$ is termed as an interval ordering relation on $I(R)$, denoted as IR.

For instance, the next five binary relation are interval ordering relations:

$$
\begin{aligned}
& \operatorname{IR}_{1}=\left\{(a, b) \mid a^{L} \leq b^{L} \text { and } a^{U} \leq b^{U}\right\} ; \\
& \operatorname{IR}_{2}=\left\{(a, b) \mid a \operatorname{IR}_{1} b \text { and } a \neq b\right\} ; \\
& \operatorname{IR}_{3}=\{(a, b) \mid m(a) \leq m(b) \text { and } w(a) \geq w(b)\} ; \\
& \operatorname{IR}_{4}=\left\{(a, b) \mid a \operatorname{IR}_{3} b \text { and } a \neq b\right\} ; \\
& \operatorname{IR}_{5}=\left\{(a, b) \mid a^{U} \leq b^{L}\right\} ;
\end{aligned}
$$

Ishibuchi and Tanaka utilized $\leq_{L U},<_{L U}, \leq_{m w}$ and $<_{m w}$ to represent the above first four relations, respectively [21]. Since $\mathrm{IR}_{1}$ and $\mathrm{IR}_{3}$ are antisymmetric, reflexive and transitive, they are partial ordering on $I(R) . \mathrm{IR}_{2}, \mathrm{IR}_{4}$, and $\mathrm{IR}_{5}$ are non-reflexive and transitive; therefore, they are quasi ordering on $I(R)$. We take the following example to illustrate the above propositions.

Example 1 Let $a=[2,3], b=[3,3.5], c=[4,4.2], d=[3,3.5], e=$ $[2.2,2.4]$.

1) Both $b \mathrm{IR}_{1} d$ and $d \mathrm{IR}_{1} b$ hold, and also it is clear that $b=d$, which means that $\mathrm{IR}_{1}$ is antisymmetric; the same is true for $\mathrm{IR}_{3}$. While for relation $\mathrm{IR}_{2}, b \mathrm{IR}_{2} d$ does not hold, which implies that $\mathrm{IR}_{2}$ does not satisfy antisymmetry; the same is true for $\mathrm{IR}_{4}$ and $\mathrm{IR}_{5}$.

2) It can be followed that $a \mathrm{IR}_{1} a$, suggesting that $\mathrm{IR}_{1}$ is reflexive; the same is true for $\mathrm{IR}_{3}$. While for $\mathrm{IR}_{2}, a \mathrm{IR}_{2} a$ does not hold, suggesting that $\mathrm{IR}_{2}$ is non-reflexive; the same is true for $\mathrm{IR}_{4}$ and $\mathrm{IR}_{5}$.

3) It can be followed that $a \mathrm{IR}_{1} b$ and $b \mathrm{IR}_{1} c$, and also it is clear that $a \mathrm{IR}_{1} c$, which implies that $\mathrm{IR}_{1}$ is transitive; the same is true for the other four relations.

4) Neither $a \mathrm{IR}_{1} e$ nor $e \mathrm{IR}_{1} a$, holds, which means that $a=[2,3]$ and $e=[2.2,2.4]$ cannot be compared by $\mathrm{IR}_{1}$, the same is true for the other four relations; as a result, all the five ordering relations are not totally ordering on $I(R)$. Particularly, more solutions cannot be distinguished by relation $\mathrm{IR}_{5}$ since its condition is very strong.

Suppose that for any $a, b \in I(R)$ and $\rho: s^{I(R) \times I(R)} \rightarrow R, \rho(a \geq b) \in[0,1]$ holds, then $\rho(a \geq b)$ is termed as a possibility degree of $a$ greater than $b$. 
For instance, Xu and Da [42] proposed the following possibility degree:

$$
p(a \geq b) \triangleq \max \left\{1-\max \left(\frac{b^{U}-a^{L}}{w(a)+w(b)}, 0\right), 0\right\} .
$$

Gao and Yang [11] reviewed a variety of definitions of possibility degrees, and summarized the possibility axiom.

\subsection{Approaches for assessing solutions of interval programming problems}

Han et al. [16] transformed an interval programming model to a deterministic single-objective one through defining the expected objectives and calculating the weight for each objective. Jiang et al. [23] converted an interval non-linear model into a deterministic bi-objective one by minimizing the midpoint and width of the objective value, and then utilized the weighted approach to transform the bi-objective one into a single-objective one. Ishibuchi and Tanaka [21] converted the original interval programming model into a deterministic multi-objective one. In the method proposed by Chanas and Kuchta [6], the original optimization model was transformed into a deterministic bi-objective one by using cut set to define an interval ordering relation. Based on the worst and the best principle, Allahdadi and Nehi [1] converted an interval objective function into a convex combination of the upper and the lower bounds of the objective value, and obtained different optimal solutions from the best case to the worst.

The above methods adopt numerical optimization methods to tackle the transformed deterministic model and most of them are for interval linear programming. Nevertheless, numerical optimization are helpless for interval nonlinear programming or complicated optimization models, such as nondifferential ones.

Jiang et al. [22] utilized the middle point and width of an interval to transform an interval nonlinear programming model to a deterministic multi-objective model. The transformed model is further converted to a non-constrained single-objective model though a linear combination of objectives and the penalty function method, and then the non-constrained model is solved by a genetic algorithm; in our previous work, we [14] also adopted the middle point and width of an interval to transform an interval multi-objective optimization problem with hybrid index to a deterministic multi-objective one, and employed NSGA-II [7] to deal with the transformed problem; Sahoo et al. [38] built a multi-objective optimization model with 
interval coefficients where objectives are system reliability and cost, transformed the constructed model into a single objective model by a scalarizing function, and tackled the transformed problem with an improved genetic algorithm; Bhunia and Samanta [4] firstly defined a variety of ordering relations and metrics of intervals to study the optimal condition for interval multi-objective programming; then, they utilized the above ordering relations and metrics to transform an interval multi-objective programming model to a deterministic single-objective one; finally, they used a hybrid tournament genetic algorithm to solve the transformed model.

The above methods transform interval programming models to deterministic ones and utilize EAs to solve the latter. It is well-understood that, for the same interval programming, different transforming methods will lead to different deterministic models. As a result, the optimal solution(s) for these deterministic models may be different as well. This means different transforming methods will lead to different optimal solution sets for the same interval programming; therefore, it is greatly difficult for a user to choose from those solution sets. In addition, some of these transforming methods introduce some parameters, such as weight for each objective, and efficient approaches are scare for specifying the values of these parameters.

Furthermore, EAs can directly tackle interval programming problems by defining interval dominance relations. Limbourg and Aponte [27] defined an interval Pareto dominance relation through an interval ordering relation; We [47] evaluated the solutions by presenting a probability dominance relation; Goh and Tan [12] calculated the probability of a solution dominating another solution and compared different solutions based on the probability; we [13] ever proposed a Pareto dominance relation based on a possibility degree of intervals; we [39] further defined the lower limit of a possibility degree, proposed a dominance relation on the basis of the lower limit and studied the properties of the corresponding Pareto sets; Karshenas et al. [24] suggested $\alpha$-degree Pareto dominance to distinguish the solutions of an interval multi-objective programming problem. Dong et al. [8] presented the scheme of interval probability dominance to distinguish different solutions. Similarly, different interval dominance relations will generate different optimal solution(s) for the same interval programming even if the same optimization algorithm is used. It can be derived from the above discussion that for both numerical and evolutionary optimization, their pivotal technique is the evaluation of solutions except for optimization algorithms. The following summarizes four approaches for assessing the solutions of model (2). 
The first one adopts relation $\mathrm{IR}_{1}$ in Eq. (3) to transform model (2) into the following multi-objective model, which maximizes the lower and upper bounds of the objective value:

$$
\begin{aligned}
& \max \left\{f^{L}(\mathbf{x}, \mathbf{c}), f^{U}(\mathbf{x}, \mathbf{c})\right\} \\
& \text { s.t. } \mathbf{x} \in \mathbf{S} \subseteq \mathbf{R}^{n},
\end{aligned}
$$

where $f^{L}(\mathbf{x}, \mathbf{c})=\left(f_{1}^{L}\left(\mathbf{x}, \mathbf{c}_{1}\right), \cdots, f_{m}^{L}\left(\mathbf{x}, \mathbf{c}_{m}\right)\right), f^{U}(\mathbf{x}, \mathbf{c})=\left(f_{1}^{U}\left(\mathbf{x}, \mathbf{c}_{1}\right), \cdots, f_{m}^{U}\right.$ $\left.\left(\mathbf{x}, \mathbf{c}_{m}\right)\right)$.

On the basis of model (5), we can deduce the following rule to distinguish solutions of model (2). Let $\mathbf{x}_{1}$ and $\mathbf{x}_{2}$ be solutions of model (2). For model (5), $\mathbf{x}_{1} \succ \mathbf{x}_{2}$, where $\succ$ is conventional Pareto dominance, then $\mathbf{x}_{1}$ is said to dominate $\mathbf{x}_{2}$; if neither $\mathbf{x}_{1}$ nor $\mathbf{x}_{2}$ dominates each other, $\mathbf{x}_{1}$ and $\mathbf{x}_{2}$ are said to be non-dominated.

The second one employs relation $\mathrm{IR}_{3}$ to transform model (2) into the following multi-objective model, which maximizes the midpoint of the objective value and minimizes its width:

$$
\begin{aligned}
& \max m(f(\mathbf{x}, \mathbf{c})), \\
& \min w(f(\mathbf{x}, \mathbf{c})), \\
& \text { s.t. } \mathbf{x} \in \mathbf{S} \subseteq \mathbf{R}^{n},
\end{aligned}
$$

where $m(f(\mathbf{x}, \mathbf{c}))=\left(m\left(f_{1}\left(\mathbf{x}, \mathbf{c}_{1}\right)\right), \cdots, m\left(f_{m}\left(\mathbf{x}, \mathbf{c}_{m}\right)\right)\right), w(f(\mathbf{x}, \mathbf{c}))=\left(w\left(f_{1}(\mathbf{x}\right.\right.$, $\left.\left.\left.\mathbf{c}_{1}\right)\right), \cdots, w\left(f_{m}\left(\mathbf{x}, \mathbf{c}_{m}\right)\right)\right)$.

Maximizing the midpoint can enhance the average performance of the objective(s), and minimizing the width can decrease the sensitivity of the objective value to the interval coefficients. In the same way, on the basis of model (6), the dominance rule between solutions of model (2) can be derived.

The third one suggested by Limbourg and Aponte [27] gives the following interval dominance relation for interval multi-objective models according to relations $\mathrm{IR}_{1}$ and $\mathrm{IR}_{2}$ of Eq. (3).

If for any $i \in\{1,2, \cdots, m\}$, all $f_{i}\left(\mathbf{x}_{2}, \mathbf{c}_{i}\right) \leq_{L U} f_{i}\left(\mathbf{x}_{1}, \mathbf{c}_{i}\right)$ hold, and there exists at least one $q \in\{1,2, \cdots, m\}$, such that $f_{q}\left(\mathbf{x}_{2}, \mathbf{c}_{q}\right)<_{L U} f_{q}\left(\mathbf{x}_{1}, \mathbf{c}_{q}\right)$ holds, i.e.

$$
\mathbf{x}_{1} \succ_{I P} \mathbf{x}_{2} \Leftrightarrow\left\{\begin{array}{l}
\forall i \in\{1,2, \cdots, m\}, \ni: f_{i}\left(\mathbf{x}_{2}, \mathbf{c}_{i}\right) \leq_{L U} f_{i}\left(\mathbf{x}_{1}, \mathbf{c}_{i}\right), \\
\exists q \in\{1,2, \cdots, m\}, \ni: f_{q}\left(\mathbf{x}_{2}, \mathbf{c}_{q}\right)<_{L U} f_{q}\left(\mathbf{x}_{1}, \mathbf{c}_{q}\right),
\end{array}\right.
$$

$\mathbf{x}_{1}$ is said to dominate $\mathbf{x}_{2}$ based on intervals. 
The fourth one presented by us [39] uses the following interval dominance relation to distinguish solutions of model (2) on the basis of the lower limit of possibility degree in Eq. (4).

For any objective function $f_{i}\left(\mathbf{x}, \mathbf{c}_{i}\right), i=1,2, \cdots, m$, if $f_{i}\left(\mathbf{x}_{1}, \mathbf{c}_{i}\right)$ dominates $f_{i}\left(\mathbf{x}_{2}, \mathbf{c}_{i}\right)$ with the possibility degree not less than $\gamma$, and there exists at least one objective function $f_{q}\left(\mathbf{x}, \mathbf{c}_{q}\right)$, such that $f_{q}\left(\mathbf{x}_{1}, \mathbf{c}_{q}\right)$ dominates $f_{q}\left(\mathbf{x}_{2}, \mathbf{c}_{q}\right)$ with the possibility degree larger than $1 / 2$, where $\gamma$ is in the range of $[1 / 2,1]$, i.e.,

$$
\mathbf{x}_{1} \succ_{\gamma} \mathbf{x}_{2} \Leftrightarrow\left\{\begin{array}{l}
\forall i \in\{1,2, \cdots, m\}, p\left(f_{i}\left(\mathbf{x}_{1}, \mathbf{c}_{i}\right) \geq f_{i}\left(\mathbf{x}_{2}, \mathbf{c}_{i}\right)\right) \geq \gamma \\
\exists q \in\{1,2, \cdots, m\}, p\left(f_{q}\left(\mathbf{x}_{1}, \mathbf{c}_{q}\right) \geq f_{q}\left(\mathbf{x}_{2}, \mathbf{c}_{q}\right)\right)>\frac{1}{2}
\end{array}\right.
$$

$\mathbf{x}_{1}$ is said to dominate $\mathbf{x}_{2}$ with the possibility degree not less than $\gamma$.

It should be noted that the third and the fourth methods can be employed to tackle interval single-objective programming models as well.

\section{Interval Dominance rules and their correlation}

Subsection 2.3 reviewed some approaches for assessing solutions of model (2). Generally speaking, the converted deterministic models usually involve multiple conflict objectives, and most of relations between intervals derived from either ordering relations or possibility degrees of intervals are not total ordering, so we borrow a terminology for multi-objective programming, i.e. dominance, to depict the relation between solutions of model (2). We call approaches for assessing solutions of model (2) as rules in the following definition for the sake of narrative.

Definition 1. All approaches for determining the dominance relations between solutions of interval programming problems are called interval dominance rules.

It is worth noting that interval dominance relations mentioned in Section 2 means dominance relations between solutions in the context of interval multi-objective programming, so they are one kind of interval dominance rules.

All rules fall into two groups. One is firstly transforming model (2) into a deterministic multi-objective model, and then assessing solutions of model (2) according to the objective value of the transformed problem, such as the first and the second methods in Subsection 2.3, denoted as rules R1 and $\mathrm{R} 2$, respectively. The other is giving a dominance relation between solutions of model (2) by directly utilizing ordering relations or possibility degrees of 
intervals, for example, the third and the fourth methods in Subsection 2.3, denoted as rules R3 and R4, respectively.

We propose the following definition about relations between interval dominance rules to further inspect their correlation.

Definition 2. Let $\mathrm{DR}_{1}$ and $\mathrm{DR}_{2}$ be two interval dominance rules to compare the solutions of model (2), and denote the dominance relation corresponding to rule $\mathrm{DR}_{u}$ as $\succ_{\mathrm{DR}_{u}}, u=1,2$.

1) For two solutions of model (2), $\mathbf{x}_{1}$ and $\mathbf{x}_{2}$, if $\mathbf{x}_{1}$ and $\mathbf{x}_{2}$ have the same dominance relation corresponding to the rules, i.e. $\mathbf{x}_{1} \succ_{\mathrm{DR}_{1}} \mathbf{x}_{2} \Leftrightarrow \mathbf{x}_{1} \succ_{\mathrm{DR}_{2}}$ $\mathbf{x}_{2}$, then rules $\mathrm{DR}_{1}$ and $\mathrm{DR}_{2}$ are equivalent;

2) for two solutions of model (2), $\mathbf{x}_{1}$ and $\mathbf{x}_{2}$, if $\mathbf{x}_{1} \succ_{\mathrm{DR}_{1}} \mathbf{x}_{2}$, then $\mathbf{x}_{1} \succ_{\mathrm{DR}_{2}}$ $\mathbf{x}_{2}$ holds; not vice versa, i.e., there exist two solutions $\mathbf{x}_{3}$ and $\mathbf{x}_{4}$, such that when $\mathbf{x}_{3} \succ_{\mathrm{DR}_{2}} \mathbf{x}_{4}, \mathbf{x}_{3}$ does not dominate $\mathbf{x}_{4}$ on the basis of rule $\mathrm{DR}_{1}$; then rule $\mathrm{DR}_{1}$ includes $\mathrm{DR}_{2}$;

3 ) If there exist two solutions of model (2), $\mathbf{x}_{1}$ and $\mathbf{x}_{2}$, such that when $\mathbf{x}_{1} \succ_{\mathrm{DR}_{1}} \mathbf{x}_{2}, \mathbf{x}_{1}$ does not dominate $\mathbf{x}_{2}$ on the basis of rule $\mathrm{DR}_{2}$; at the same time, there exist two other solutions of model (2), $\mathbf{x}_{3}$ and $\mathbf{x}_{4}$, such that when $\mathbf{x}_{3} \succ_{\mathrm{DR}_{2}} \mathbf{x}_{4}, \mathbf{x}_{3}$ does not dominate $\mathbf{x}_{4}$ on the basis of rule $\mathrm{DR}_{1}$; then rules $\mathrm{DR}_{1}$ and $\mathrm{DR}_{2}$ are exclusive.

Definition 2 describes the correlation between rules through equivalence, inclusion, and exclusion. More specifically, the correlations between interval dominance rules are from strong to weak w.r.t. the above relations; particularly, when two rules are equivalent, their correlation is the strongest.

We take the following example to show the correlation between rules defined by Definition 2 .

Example 2. For the rules R1, R2, R3 and R4, given in Subsection 2.3, the following conclusions hold.

1) Rules R1 and R3 are equivalent;

2) rules $\mathrm{R} 1$ and $\mathrm{R} 2$ are exclusive, so do $\mathrm{R} 2$ and $\mathrm{R} 4$;

3) when $\gamma=0.5$ in R4, R1 includes R4; when $\gamma>0.5$, rules R1 and R4 are exclusive.

Please see the proof of this example in Appendix A.

It is a bit difficult to determine the relationship between rules by using Definition 2 as shown from the proof in Appendix A. However, it can be known from Definition 2 that, for any solution set of model (2), if two interval dominance rules are equivalent, the non-dominated solution sets corresponding to the two rules must be the same; if the two rules is inclusive, the non-dominated solution sets corresponding to them are inclusive as well; 
if the two rules are exclusive, the non-dominated solution sets corresponding to them also mutually exclude. Conversely, the correlation between interval dominance rules can also be inspected via the relationship between nondominated solution sets corresponding to them. On the basis of the above discussion, we give the following equivalent definition of the correlation between interval dominance rules.

Definition 3. Let $\mathrm{DR}_{1}$ and $\mathrm{DR}_{2}$ be interval dominance rules for comparing solutions of model (2), $X$ be any solution set of model (2), and $X_{1}^{*}$ and $X_{2}^{*}$ be the non-dominated solution sets of $X$ corresponding to the two rules, respectively. If $X_{1}^{*}=X_{2}^{*}$, rules $\mathrm{DR}_{1}$ and $\mathrm{DR}_{2}$ are equivalent. If $X_{1}^{*} \subset X_{2}^{*}$, rule $\mathrm{DR}_{2}$ includes $\mathrm{DR}_{1}$; otherwise, rules $\mathrm{DR}_{1}$ and $\mathrm{DR}_{2}$ mutually exclude.

The usage of Definition 2 requests users to be very familiar with various interval dominance rules and theoretically analyze their intrinsic relationships. It is neither possible nor necessary for most users, for they only utilize these rules without learning more about their intrinsic qualities. Different from Definition 2, utilizing Definition 3 to study the correlation between rules just needs to get non-dominated solution sets corresponding to these rules. As there are numerous approaches for generating non-dominated solution sets, Definition 3 is more pragmatic.

It can be followed from Definition 3 that, if there exists a solution set of model (2), such that non-dominated solution sets corresponding to two interval dominance rules are unequal; these two rules must be nonequivalent. Nevertheless, computer cannot check whether non-dominated solution sets based on two interval dominance rules are equal for any solution set due to its limit computational capability; therefore, we utilize the law of large numbers to derive the following proposition to discern the equivalent relation between interval dominance rules.

Proposition 1. Let $\mathrm{DR}_{1}$ and $\mathrm{DR}_{2}$ be two interval dominance rules for comparing the solutions of model (2). If non-dominated solution sets corresponding to them for a variety of solution sets of model (2) are equal, the two rules are equivalent.

Algorithm 1 depicts the pseudocode for discerning the equivalent relationship between interval dominance rules on the basis of Proposition 1. At the start, a solution set $P$ is randomly created, and the number of non-dominated solution sets based on two rules being equal is set as 0 , as shown in lines 3 and 4; then, non-dominated solution sets, $P_{1}$ and $P_{2}$, are produced by the two rules, as shown in lines 5 to 18, where dominated solutions are removed from set $P$, and the rest of solutions forms a non-dominated solution set; fi- 
nally, whether the numbers of solutions consisted in two non-dominated sets are equal is checked; if they are unequal, the two rules are nonequivalent; if they are equal, whether two non-dominated sets are equal is further checked; if yes, the number of non-dominated solution sets being equal is added by 1 ; otherwise, two rules are nonequivalent. For $L$ randomly created solution sets, only when non-dominated solution sets corresponding to the two rules are equal, i.e., the number of non-dominated solution sets being equal is $L$, the two rules are equivalent.

The correlation, in particular, the inclusive and exclusive relationship between interval dominance rules theoretically reveals that different interval dominance rules will generate different optimal solution(s) for the same interval programming, which means that they are somewhat subjective. When a user adopts an interval dominance rule to assess solutions of an interval programming problem, other users may dissatisfy with the final optimal solution(s) and scepticize over its(their) objectivity and rationality. If a variety of rules or approaches are simultaneously adopted to assess solutions of the problem, diverse and complemental evaluations for the solutions of the model can be reached; further, if we assemble these evaluations, which is similar to group decision making [35, 44], final optimal solution(s) will be accepted by more users. This means that the users need not struggle with the conversions of interval programming models or the comparison between intervals so that they can focus on the design of optimization algorithms. In this way, interval programming can be applied to more real-world situations. In view of this, the following section will suggest an approach for assembling interval dominance rules.

\section{An Ensemble framework for interval dominance rules}

This section focuses on developing an ensemble framework for interval dominance rules. To this end, it is imperative to answer the following two questions: one is whether different dominance rules can generate diverse optimal solution sets; the other is how to assemble these rules. Based on the above considerations, the framework is divided into two modules. One is reducing any redundant interval dominance rules to derive a reduced rule set, which can be accomplished before optimization. The other is assembling the reduced dominance rules, which can be embedded into any optimization algorithm and whose role is comprehensively evaluating solutions during optimization. The method for reducing the dominance rules is expounded as 


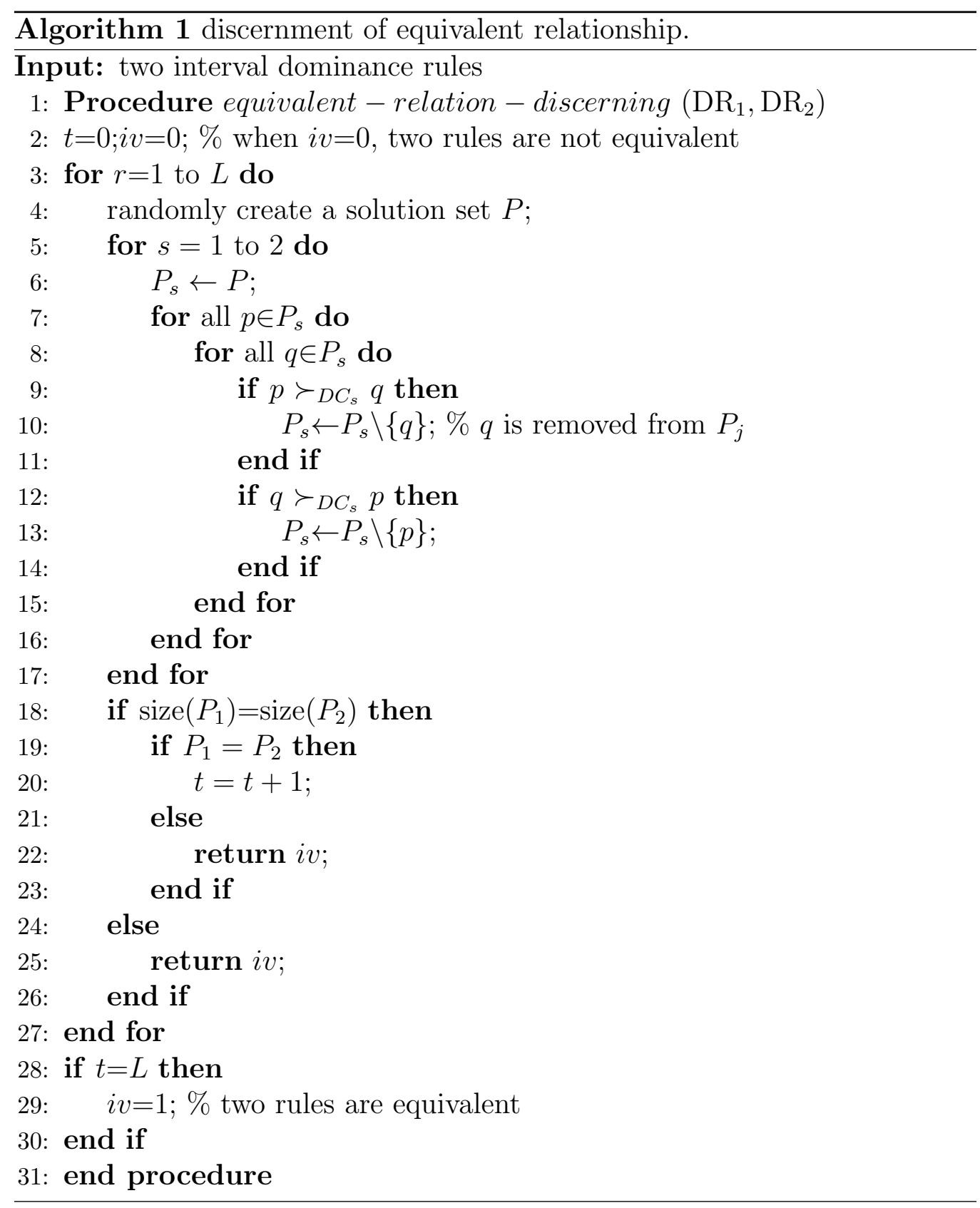


follows.

\subsection{Reducing interval dominance rules}

Assume that $M$ interval dominance rules, called a rule set, are employed to tackle an interval programming model. It should be noted that sometimes different dominance rules may generate the same optimal solution(s). The ensemble aims to objectively and comprehensively assess solutions of the model, and a lot of same assessments will weaken the effect of the other assessments, which has a negative influence on the fairness of assembling. In this way, the final optimal solution(s) can only be satisfied by a small number of users. Therefore, it is prerequisite to remove redundant rule(s) from the rule set so as to produce diverse assessments.

We will discuss the impact of different relationships between interval dominance rules on the sorting of solutions in order to develop a method to reduce the rule set.

The first case is multiple rules being equivalent. It can be followed from Definition 3 that the sorting of any solution set corresponding to them are totally same, suggesting that their combination is insignificant. Therefore, just one of them should be remained.

The second case is rule $\mathrm{DR}_{v}$ including $\mathrm{DR}_{w}, v, w \in 1,2, \cdots, M$, and $v \neq w$. In this case, the non-dominated solution set corresponding to rule $\mathrm{DR}_{v}$ includes that corresponding to $\mathrm{DR}_{w}$, which means that the rank of solutions whose rank is 1 corresponding to $\mathrm{DR}_{w}$ is also 1 corresponding to $\mathrm{DR}_{v}$; however, the ranks of other solutions corresponding to two rules might not be the same. Additionally, it usually needs to use other indicators to evaluate solutions with the same rank, and different indicators will produce different sorting of a solution set. Therefore, the two rules with inclusive relationship will generate different sorting, and the rules with inclusive relationship should be kept.

The last case is rules being exclusive. Under this situation, they will certainly generate different non-dominated solution sets and there is no denying that they will produce different sorting; therefore, exclusive rules are beneficial to enhance the fairness of assembling and should be kept.

In conclusion, the reduction strategy of rules is removing equivalent rules that have the strongest correlation, and remaining inclusive and exclusive rules. Based on Algorithm 1, Algorithm 2 depicts the reduction strategy of interval dominance rules. 


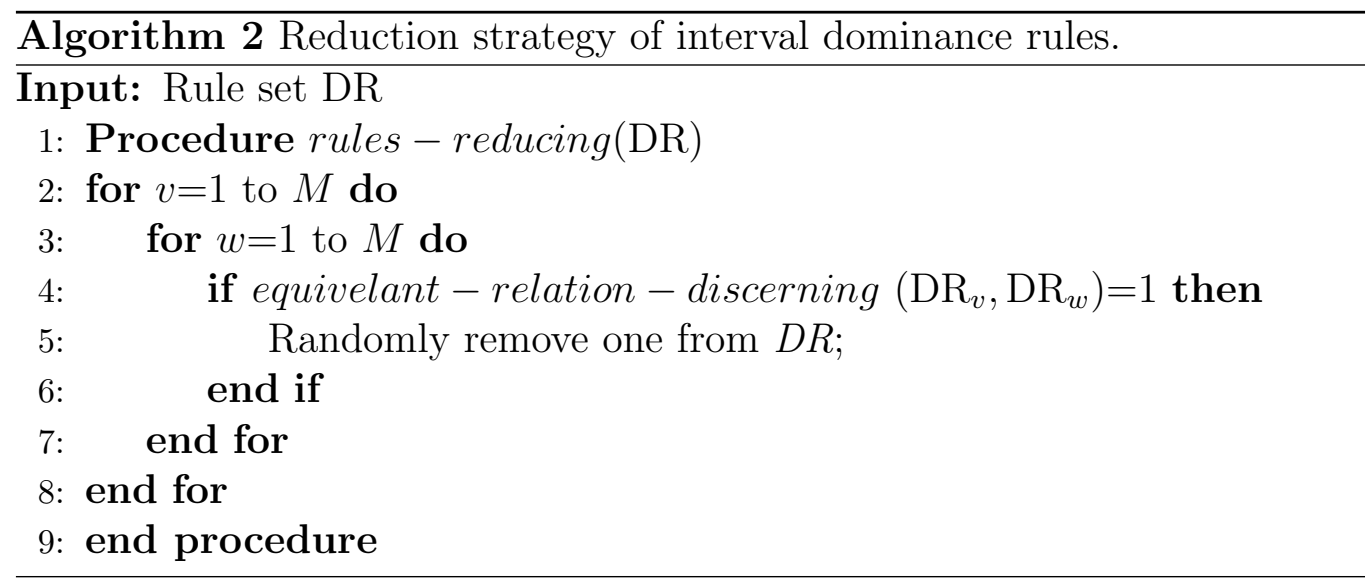

\subsection{An ensemble dominance relation}

This subsection suggests an ensemble dominance relation to deal with interval programming models on the basis of the reduced rule set in Subsection 4.1. Suppose that there are $Q$ rules in the reduced rule set, denoted as $\mathrm{DR}_{1}$, $\mathrm{DR}_{2}, \cdots, \mathrm{DR}_{Q}$, respectively. They can be assembled by firstly comparing two solutions based on each of them, then integrating the comparison results on two solutions corresponding to all rules, and finally ordering the solutions by the integrated comparison. Such an ensemble strategy is called integrating first and ordering last. Additionally, there is an alternative strategy, such as sorting first and integrating last, as well as possible others [35, 44]. As a result, the ensemble of interval dominance rules has rich prospects. However, it is worth noting that all of the above approaches assemble the assessments for solutions rather than optimization algorithms.

We put forward an ensemble dominance relation as follows as an example by applying the integrating first and ordering last strategy.

Definition 4. Let $\mathbf{x}_{1}$ and $\mathbf{x}_{2}$ be solutions of model (2), the indicator $I_{h}\left(\mathbf{x}_{1}, \mathbf{x}_{2}\right)$ represents the result by employing the $h$-th rule to compare $\mathbf{x}_{1}$ and $\mathbf{x}_{2}$, i.e.,

$$
I_{h}\left(\mathbf{x}_{1}, \mathbf{x}_{2}\right)=\left\{\begin{array}{l}
1, \mathbf{x}_{1} \succ_{\mathrm{DR}_{h}} \mathbf{x}_{2} \\
-1, \mathbf{x}_{2} \succ_{\mathrm{DR}_{h}} \mathbf{x}_{1} \\
0, \text { others }
\end{array}\right.
$$

Let us define an ensemble indicator, $I\left(\mathbf{x}_{1}, \mathbf{x}_{2}\right)$, to represent the assembled 
order relationship between $\mathbf{x}_{1}$ and $\mathbf{x}_{2}$ as $I\left(\mathbf{x}_{1}, \mathbf{x}_{2}\right)=\sum_{h=1}^{Q} \omega_{h} I_{h}\left(\mathbf{x}_{1}, \mathbf{x}_{2}\right)$, where $\omega_{h} \geq 0$ and $\sum_{h=1}^{Q} \omega_{h}=1$; when $I>0.5, \mathbf{x}$ is said to ensemble dominate $\mathbf{x}_{2}$, denoted as $\mathbf{x}_{1} \succ_{e d} \mathbf{x}_{2}$; when $I<0.5, \mathbf{x}_{2}$ is said to ensemble dominate $\mathbf{x}_{1}$, denoted as $\mathbf{x}_{2} \succ_{e d} \mathbf{x}_{1}$; otherwise, $\mathbf{x}_{1}$ and $\mathbf{x}_{2}$ are ensemble non-dominated.

Note: 1) $\mathbf{x}_{2} \succ_{\mathrm{DR}_{h}} \mathbf{x}_{1}$ in Eq.(9) means that $\mathbf{x}_{2}$ dominates $\mathbf{x}_{1}$ on the basis of rule $\mathrm{DR}_{h}$ that may be related to an ordering relation or a possibility degree of intervals, such as Eq.(8), so we use 1,-1 and 0 to represent the dominance relation between $\mathbf{x}_{1}$ and $\mathbf{x}_{2}$. The possibility degree can certainly be employed to assess the solutions directly, which will be another novel ensemble approach.

2) $\omega_{h}$ is the weight of the $h$-th interval dominance rule, which is just like the weight of an expert in a group decision making and reflects the importance of a rule in an ensemble dominance relation. When users have little knowledge about the difference between interval dominance rules, $\omega_{h}$ can be set as $\frac{1}{Q}$, suggesting that the roles of all the rules are the same when using the ensemble dominance relation to assess the solutions; if users prefer to the $h$-th rule, they can assign a higher value to $\omega_{h}$, suggesting that the $h$-th rule plays an important role in the assessment of the solutions. Additionally, the other preference types, such as hesitant fuzzy linguistic preference relations [43], can also used to assemble the interval dominance rules.

We take the following example to show the usage of Definition 4 and reveal the impacts of the ensemble dominance relation and the weights on the optimal solutions.

Example 3 Consider a numerical interval linear programming example as follows [45]:

$$
\begin{aligned}
& \max f=[3,3.5] x_{1}-[1,1.2] x_{2} \\
& \text { s.t. }[1,1.1] x_{1}+[1.6,1.8] x_{2} \leq[11.6,12] \\
& {[3,4] x_{1}-[2,3] x_{2} \leq[5,7]} \\
& x_{1}, x_{2} \geq 0
\end{aligned}
$$

Table 1 lists some solutions of problem (10) and corresponding objective values.

1) We first investigate the relation between $\mathbf{x}_{2}$ and $\mathbf{x}_{3}$ on the basis $R 1$, $\mathrm{R} 2$ and $\mathrm{R} 4$ given in Subsection 2.3. It can be followed that $\mathbf{x}_{2}$ and $\mathbf{x}_{3}$ are non-dominated on the basis of R1, $\mathbf{x}_{2}$ dominates $\mathbf{x}_{3}$ on the basis of R2, and $\mathbf{x}_{2}$ 
Table 1: Some solutions of problem (10) and corresponding objective values

\begin{tabular}{cccccc}
\hline solution & $\mathbf{x}_{1}$ & $\mathbf{x}_{2}$ & $\mathbf{x}_{3}$ & $\mathbf{x}_{4}$ & $\mathbf{x}_{5}$ \\
\hline value & $(2,0)$ & $(2,1)$ & $(3,4)$ & $(3,3.5)$ & $(4,5)$ \\
objective value & {$[6,7]$} & {$[4.8,6]$} & {$[4.2,6.5]$} & {$[4.8,7]$} & {$[6,9]$} \\
\hline
\end{tabular}

and $\mathbf{x}_{3}$ are non-dominated on the basis of $\mathrm{R} 4$ when $\gamma=0.8$, which indicates that different interval dominance rules likely reach different assessments of solutions.

2) If $\omega_{h}=1 / 3, h=1,2,3$ in Eq.(9), $I\left(\mathbf{x}_{2}, \mathbf{x}_{3}\right)=1 / 3$. Therefore, $\mathbf{x}_{2}$ and $\mathbf{x}_{3}$ are ensemble non-dominated according to Definition 4 , which means that the proposed ensemble dominance relation integrates assessments of three interval dominance rules. Whereas if $\omega_{1}=0.2, \omega_{2}=0.6$ and $\omega_{3}=0.2$ in Eq.(9), $I\left(\mathbf{x}_{2}, \mathbf{x}_{3}\right)=0.6$. So $\mathbf{x}_{2}$ ensemble dominates $\mathbf{x}_{3}$ according to Definition 4. The setting of weights implies that the user prefers to the second interval dominance relation; thereby comprehensive assessment will more accord with the assessment of $\mathrm{DR}_{2}$.

3) For solutions $\mathbf{x}_{1}$ and $\mathbf{x}_{5} \cdot \mathbf{x}_{5}$ dominates $\mathbf{x}_{1}$ on the basis of R1 and they are non-dominated on the basis of $\mathrm{R} 2$ and $\mathrm{R} 4$. If $\omega_{h}=1 / 3, h=1,2,3$ in Eq.(9), they are ensemble non-dominated. If R3 that is equivalent with R1 is not deleted from the rule set and there are other two interval dominance rules that are equivalent with $\mathrm{R} 1, \mathbf{x}_{5}$ also dominates $\mathbf{x}_{1}$ on the basis of $\mathrm{R} 1$ and the other two equivalent rules. In this case, if $\omega_{h}=1 / 6, h=1, \cdots, 6$ in Eq.(9), there is no doubt that $\mathbf{x}_{5}$ ensemble dominates $\mathbf{x}_{1}$. It is obvious that the result is determined by multiple equivalent rules, which is unfair and unfavorable for the ensemble. Therefore, it is quite necessary to reduce the redundant rules so as to improve the ensemble effectiveness.

4) For the solution set $X=\left\{\mathbf{x}_{1}, \mathbf{x}_{2}, \mathbf{x}_{3}, \mathbf{x}_{4}, \mathbf{x}_{5}\right\}$, its optimal solution sets on the basis of R1, R2 and R4 are $\left\{\mathbf{x}_{5}\right\},\left\{\mathbf{x}_{1}, \mathbf{x}_{2}, \mathbf{x}_{4}, \mathbf{x}_{5}\right\}$ and $\left\{\mathbf{x}_{1}, \mathbf{x}_{5}\right\}$, respectively, when $\gamma=0.8$ in R4. If we use $\mathrm{IR}_{5}$ in Eq. (3) to compare the objective values, the optimal solution set will be $\left\{\mathbf{x}_{1}, \mathbf{x}_{3}, \mathbf{x}_{4}, \mathbf{x}_{5}\right\}$ since the strong condition of $\mathrm{IR}_{5}$ makes more solutions incomparable. If $\omega_{h}=1 / 3, h=1,2,3$ in Eq.(9), the optimal solution set on the basis of the proposed ensemble dominance relation is $\left\{\mathbf{x}_{1}, \mathbf{x}_{5}\right\}$, which indicates that when all the interval dominance rules have the same importance, the proposed ensemble dominance relation can mirror assessments of most interval dominance rules. If R1, R2 and R4 correspond to three users, and the corresponding optimal solution sets are their decision results, the optimal solution set on the basis 
of the proposed ensemble dominance relation is clearly the set of most users' decision results, suggesting that the proposed ensemble dominance can achieve optimal solution(s) satisfied by more users. Additionally, it can be seen that there is usually no unique optimal solution for an interval programming problem due to the particularity of interval uncertainty, even for single-objective interval programming. This means that users should choose their most favorable one from multiple optimal solutions, which belongs to the problem of sorting intervals or interval multi-criteria decision making, and will be our future research topics.

Example 3 shows that the proposed ensemble dominance can be employed to assess solutions of any interval programming problem no matter whether the objective function(s) is/are linear or not. So the conclusions of Example 3 hold as well for interval nonlinear programming, which will be demonstrated in Subsection 5.3 for both interval single-objective and multi-objective nonlinear programming.

The following proposition can be easily derived from Eq. (9).

Proposition 2. Given that $\mathbf{x}_{1}$ and $\mathbf{x}_{2}$ are solutions of model (2), then $I\left(\mathbf{x}_{1}, \mathbf{x}_{2}\right)+I\left(\mathbf{x}_{2}, \mathbf{x}_{1}\right)=0$.

Proof. It can be followed from Eq. (9) that $I_{h}\left(\mathbf{x}_{1}, \mathbf{x}_{2}\right)+I_{h}\left(\mathbf{x}_{2}, \mathbf{x}_{1}\right)=0$. So

$I\left(\mathbf{x}_{1}, \mathbf{x}_{2}\right)+I\left(\mathbf{x}_{2}, \mathbf{x}_{1}\right)=\sum_{h=1}^{Q} \omega_{h} I_{h}\left(\mathbf{x}_{1}, \mathbf{x}_{2}\right)+\sum_{h=1}^{Q} \omega_{h} I_{h}\left(\mathbf{x}_{2}, \mathbf{x}_{1}\right)=\sum_{h=1}^{Q} \omega_{h}\left(I_{h}\left(\mathbf{x}_{1}, \mathbf{x}_{2}\right)+\right.$ $\left.I_{h}\left(\mathbf{x}_{2}, \mathbf{x}_{1}\right)\right)=0$, which completes the proof.

Proposition 2 implies that $\mathbf{x}_{1}$ ensemble dominating $\mathbf{x}_{2}$ and $\mathbf{x}_{2}$ ensemble dominating $\mathbf{x}_{1}$ cannot simultaneously hold. As a consequence, Definition 4 is rational.

\subsection{Complexity of the framework}

For two modules of the proposed framework, their complexities will be discussed in this subsection.

Proposition 3. Assume that there are $M$ interval dominance rules in the rule set, and $L$ solution sets, each of which involves $N$ solutions, are randomly created; then the complexity of reducing dominance rules is $O\left(L M^{2} N^{2}\right)$.

Proof. The discussion is divided into two steps. The first step discusses the complexity of checking whether any pair of rules are equivalent; the second one investigates the complexity of reducing dominance rules. In the first step, the worst case is that $N$ solutions are non-dominated corresponding to the two rules. In this case, the complexity of obtaining two non-dominated 
solution sets is $O\left(2 N^{2}\right)$, and the two non-dominated solution sets are absolutely equal. The worst case is the above steps are repeated $L$ times. Therefore, in the worst case, the complexity of Algorithm 1 is $O\left(2 L N^{2}\right)$. In the second one, the worst case is that any pair of rules are nonequivalent, then Algorithm 2 should run $C_{M}^{2}$ times. To sum up, in the worst case, the complexity of reducing rules is $O\left(L M^{2} N^{2}\right)$.

It can be seen from Proposition 3 that the running time of reducing rules polynomially increases with the increase of the number of rules and the number of any solution sets. As the module can be accomplished before optimization, there is no impact on the optimizer. Additionally, if employing Definition 2 to discern the relationship between rules, reducing a rule set will not prolong the optimizer.

Proposition 4. Assume that there are $Q$ rules in the reduced rule set, and $Q \leq M$, the complexity of determining the ensemble dominance relation is $O(m Q)$, where $m$ is the number of objectives of model (2).

Proof. When employing Algorithm 2 to determine the ensemble dominance relation between solutions, the dominance indicators based on $Q$ rules are firstly calculated respectively; in this case, it is necessary to perform $m Q$ comparison operators; then the relation is derived by calculating the ensemble dominance indicator; in this case, it is necessary to perform one comparison operator. Thereby, the complexity of determining the ensemble dominance relation is $O(m Q)$.

It can be implied from Proposition 4 that the running time of comparing two solutions corresponding to the proposed ensemble dominance linearly increases with the increase of the number of the reduced rules, indicating that, if utilizing it to tackle an interval programming model, the time for evaluating the solutions will be long.

A method for assembling interval dominance rules is proposed and illustrated in this subsection. The proposed ensemble dominance will be tested on three groups of experiments in the next section.

\section{Experiments}

The experiments are divided into three groups to demonstrate the rationality and effectiveness of the proposed framework. The first one shows the effectiveness of Algorithm 1 by utilizing it to discern the correlations between any pair of six previous interval dominance rules; the second one discloses the intrinsic quality of the proposed ensemble dominance by assembling the 
Table 2: The standard deviations

\begin{tabular}{lc}
\hline \multicolumn{1}{c}{ problems } & $\delta$ \\
\hline $\begin{array}{c}\text { single-objective } \\
\text { bi-objective }\end{array}$ & $\left(0.1\left|\sin \left(10 \pi \sum_{i} x_{i}\right)\right|, 0.1\left|\sin \left(20 \pi \sum_{i} x_{i}\right)\right|\right)$ \\
\hline
\end{tabular}

six rules and surveying the relation between the non-dominated solution set based on the ensemble dominance relation and those based on the reduced rules; the third one assesses the performance of the proposed framework by embedding it into an evolutionary interval multi-objective optimization algorithm and comparing the algorithm with three other ones, each of which adopt a single interval dominance rule. The implementation environmen$\mathrm{t}$ is as follows: Pentium(R) Dual-Core CPU, 4G RAM, windows XP and Matlab R2008a. The EA embedded by the proposed ensemble framework is IP-MOEA [27], in which the traditional dominance relation and the crowding distance of NSGA-II [7] are replaced by an interval dominance relation and the hyper-volume contribution of an individual, respectively.

\subsection{Interval test models}

Two single-objective test models, i.e., Rosenbrock and Sphere, and four bi-objective test models, i.e., ZDT1, ZDT2, ZDT4 and ZDT6 [7] are taken into account in the experiments. The number of decision variables of each model is 30. The ranges of decision variables of Rosenbrock and Sphere are $[-2.048,2.048]$ and $[-100,100]$, respectively; the range of decision variables of bi-objective test models is $[0,1]$.

Since all the above models are minimization problems and their objective values are exact, the objective values are firstly normalized, and the method for transforming minimization problems to maximization ones [27] as well as the standard deviation $\sigma$ [15] for making them intervals are adopted. The standard deviations are listed in Table 2. The corresponding interval models are denoted as Rosenbrock $_{I}$, Sphere $_{I}, \mathrm{ZDT}_{I} 1, \mathrm{ZDT}_{I} 2, \mathrm{ZDT}_{I} 4$ and $\mathrm{ZDT}_{I} 6$, respectively.

\subsection{The effectiveness of discerning equivalent relation}

For six previous interval dominance rules, the correlations between any pair of rules are investigated by Algorithm 1 in this group of experiments. These six interval dominance rules are R1, R2, R3 and R4, in which $\gamma=0.5$, 
Table 3: Correlations between any pair of six interval dominance rules

\begin{tabular}{ccccccc}
\hline & R1 & R2 & R3 & R4 & R5 & R6 \\
\hline R1 & 1 & 0 & 1 & 0 & 0 & 0 \\
R2 & 0 & 1 & 0 & 0 & 0 & 0 \\
R3 & 1 & 0 & 1 & 0 & 0 & 0 \\
R4 & 0 & 0 & 0 & 1 & 0 & 0 \\
R5 & 0 & 0 & 0 & 0 & 1 & 0 \\
R6 & 0 & 0 & 0 & 0 & 0 & 1 \\
\hline
\end{tabular}

in Subsection 2.3, and two others presented by us [13, 47], denoted as R5 and R6, respectively. R5 and R6 are described in Appendix B.

A solution set involving 200 solutions is firstly randomly created so as to discern the correlation between any pair of dominance rules; then, objective values are calculated by a model, for example $\mathrm{ZDT}_{I} 1$; finally, the correlation between rules is discerned by checking whether their non-dominated solution sets are equal. The above process is independently run 20 times.

Table 3 reports the correlations between any pair of six interval dominance rules, where 0 and 1 represent non-equivalence and equivalence, respectively. It can be followed from Table 3 that any pair of others are nonequivalent except that R1 and R3 are equivalent. The conclusion about R1, R2, R3 and R4 is coincident with Example 2, which indicates that Algorithm 1 can effectively discern the correlation between interval dominance rules. In addition, the reduced rule set of the six interval dominance rules, i.e., $\{\mathrm{R} 1$, R2, R4, R5, R6\}, can be deduced via Table 3.

\subsection{Rationality of ensemble dominance}

The rationality of the proposed ensemble dominance is inspected in this group of experiments for the reduced rule set derived in the first one. For this purpose, a solution set involving 200 solutions is firstly created; then, the objective values of each test model are calculated, and R1, R2, R4, R5, R6 as well as their ensemble dominance relation, denoted as ED, are used to produce the corresponding non-dominated solution sets; finally, the relationships between these non-dominated sets are analyzed. Here, assume that we have little knowledge about each interval dominance rule, and therefore, all $\omega_{h}$ in Definition 4 are set as $0.2, h=1,2, \cdots, 5$.

For each test model, Table 4 reports the optimal solution(s) of the same solution set based on different interval dominance rules, where the number 
Table 4: Optimal solution(s) corresponding to five interval dominance rules and their ensemble dominance relation

\begin{tabular}{|c|c|c|c|c|c|c|}
\hline & Rosenbrock $_{I}$ & Sphere $_{I}$ & $\mathrm{ZDT}_{I} 1$ & $\mathrm{ZDT}_{I} 2$ & $\mathrm{ZDT}_{I} 4$ & $\mathrm{ZDT}_{I} 6$ \\
\hline$\overline{\mathrm{R} 1}$ & $\begin{array}{l}58,145, \\
177,198\end{array}$ & 135 & $\begin{array}{c}75,80,90, \\
146,167\end{array}$ & $\begin{array}{c}30,51, \\
129,185\end{array}$ & $6,117,122$ & $\begin{array}{c}42,98, \\
131,175\end{array}$ \\
\hline $\mathrm{R} 2$ & $\begin{array}{c}58,186 \\
198\end{array}$ & $\begin{array}{c}44,94, \\
135,138\end{array}$ & 183 & 46,185 & 59 & 163 \\
\hline $\mathrm{R} 4$ & 58 & $\begin{array}{c}75,80,90 \\
135,146, \\
167\end{array}$ & $16,56,75$ & $\begin{array}{l}30,51,62 \\
129,152 \\
178,185\end{array}$ & $\begin{array}{c}6,12 \\
117,122 \\
136\end{array}$ & $\begin{array}{c}42,98, \\
131,173, \\
175\end{array}$ \\
\hline $\mathrm{R} 5$ & 198 & $\begin{array}{c}70,75,80 \\
90,135, \\
146,167\end{array}$ & $16,56,62$ & $\begin{array}{c}30,51,62 \\
129,152 \\
178,185\end{array}$ & $\begin{array}{c}6,12 \\
117,122 \\
136\end{array}$ & $\begin{array}{c}42,98, \\
131 \\
175\end{array}$ \\
\hline R6 & 58 & 135 & $\begin{array}{c}16,56,75 \\
80,90, \\
146,167\end{array}$ & $\begin{array}{c}30,51,62 \\
129,152 \\
178,185\end{array}$ & $\begin{array}{c}6,12 \\
117,122 \\
136\end{array}$ & $\begin{array}{c}42,98, \\
131,173, \\
175\end{array}$ \\
\hline ED & 58,198 & 135 & $16,56,75$ & $\begin{array}{c}30,51,62 \\
129,152 \\
178,185\end{array}$ & $\begin{array}{c}6,12 \\
117,122 \\
136\end{array}$ & $\begin{array}{c}42,98, \\
131,175\end{array}$ \\
\hline
\end{tabular}


Table 5: Parameter settings

\begin{tabular}{cccccc}
\hline $\begin{array}{c}\text { population } \\
\text { size }\end{array}$ & $\begin{array}{c}\text { maximal } \\
\text { generation }\end{array}$ & $\begin{array}{c}\text { SBX } \\
\text { probability }[7]\end{array}$ & $\begin{array}{c}\text { Polynomia mutation } \\
\text { probability }[7]\end{array}$ & $\begin{array}{c}\eta_{c} \\
{[7]}\end{array}$ & $\begin{array}{l}\eta_{m} \\
{[7]}\end{array}$ \\
\hline 50 & 100 & 0.9 & $1 / 30$ & 20 & 20 \\
\hline
\end{tabular}

represents the ordinal number of a solution in the solution set.

It can be derived from Table 4 that the non-dominated solution set based on the proposed ensemble dominance is neither the union of optimal solution(s) based on the others nor their intersection, but rather the one composed by optimal solution(s) according to most of the dominance rules, which mirrors the simple idea of collective intelligence. The proposed ensemble dominance is thus rational.

\subsection{Applications to interval multi-objective test models}

$\mathrm{R} 1, \mathrm{R} 2, \mathrm{R} 4$ and their ensemble dominance relation, in which all $\omega_{h}, h=$ $1,2,3$ in Definition 4 are set as $1 / 3$, are employed to deal with four interval bi-objective models, i.e., $\mathrm{ZDT}_{I} 1, \mathrm{ZDT}_{I} 2, \mathrm{ZDT}_{I} 4$ and $\mathrm{ZDT}_{I} 6$, in this group of experiments to evaluate the performance of the proposed ensemble framework. Four rules are incorporated into IP-MOEA [27], i.e., all optimization algorithms are the same except for dominance relations in non-dominated sorting. Each algorithm is independently run 20 times, and the means of these results are calculated.

All algorithms use the same parameter settings, as shown in Table 5.

The following three performance indicators are adopted to evaluate the performances of different optimizers.

1) The worst hypervolume [27] (H metric, for short). The larger the value of the worst hypervolume of the final Pareto front is, the closer the final front to the true Pareto front and the better the distribution of points along the front are. Both reference points are set as $(-1,-1)^{T}$.

2) Imprecision [27] (I metric, for short), which mirrors the uncertainty of the final Pareto front. The smaller the imprecision is, the more exact the final front and the better the front is.

3) CPU time. The shorter the CPU time of an optimizer is, the higher its efficiency is.

Figures 1 and 2 plot the curves of the $\mathrm{H}$ and I metrics w.r.t. the number of generations, respectively. It can be seen from the figures that: 

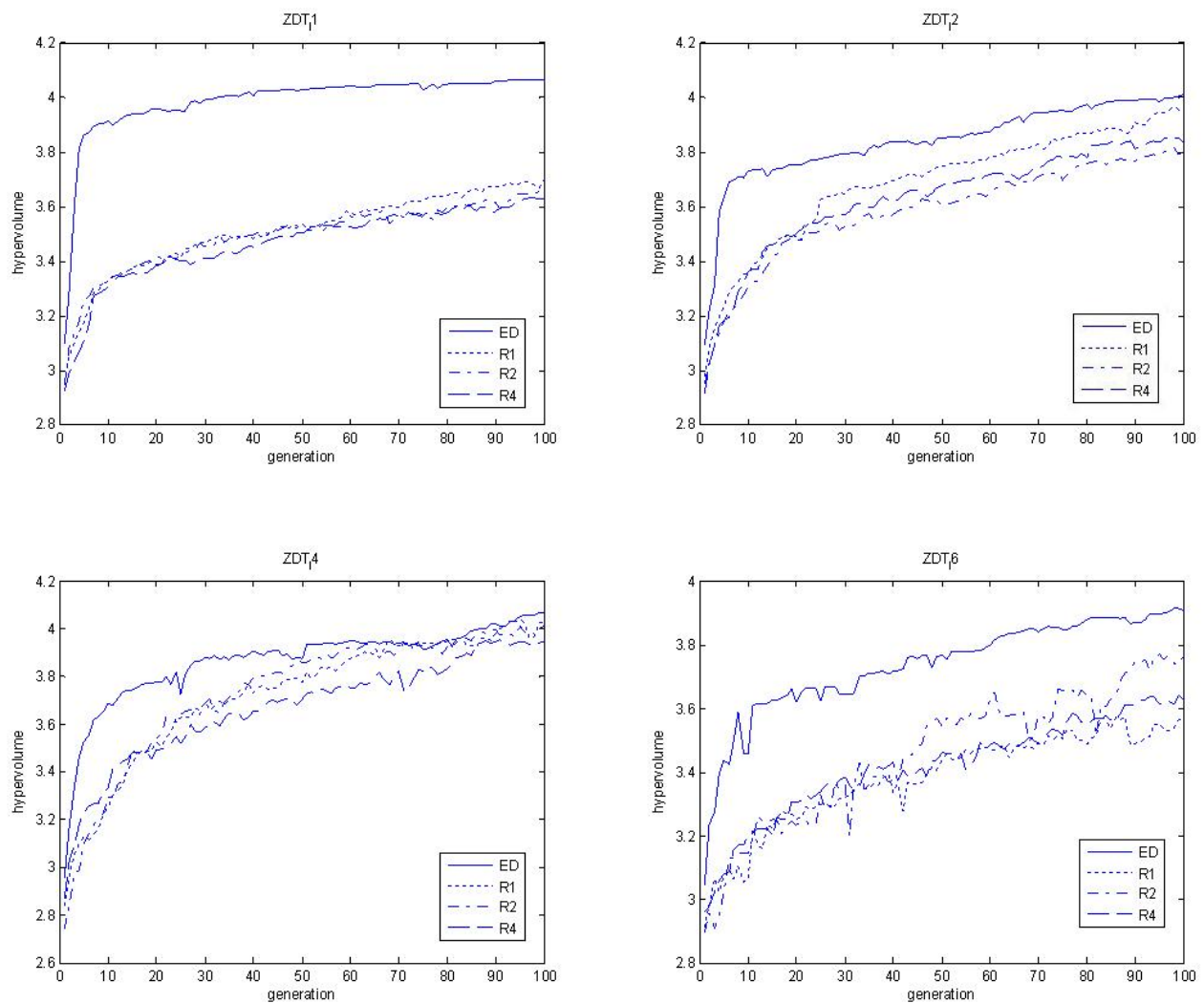

Figure 1: Curves of $\mathrm{H}$ metric w.r.t. number of generations

1) for the same generation, the value of $\mathrm{H}$ metric obtained by the proposed ensemble dominance relation is bigger than those obtained by other rules, which means that the proposed framework can generate a Pareto front closest to the true one.

2) for the same generation, the value of I metric obtained by the proposed ensemble dominance relation is far smaller than those obtained by other rules, which indicates that the proposed framework can produce a Pareto front with the least uncertainty.

Table 6 reports the values of three performance indicators obtained by different dominance rules. The superscript means that the datum with it is significantly inferior to that obtained by the proposed ensemble dominance 

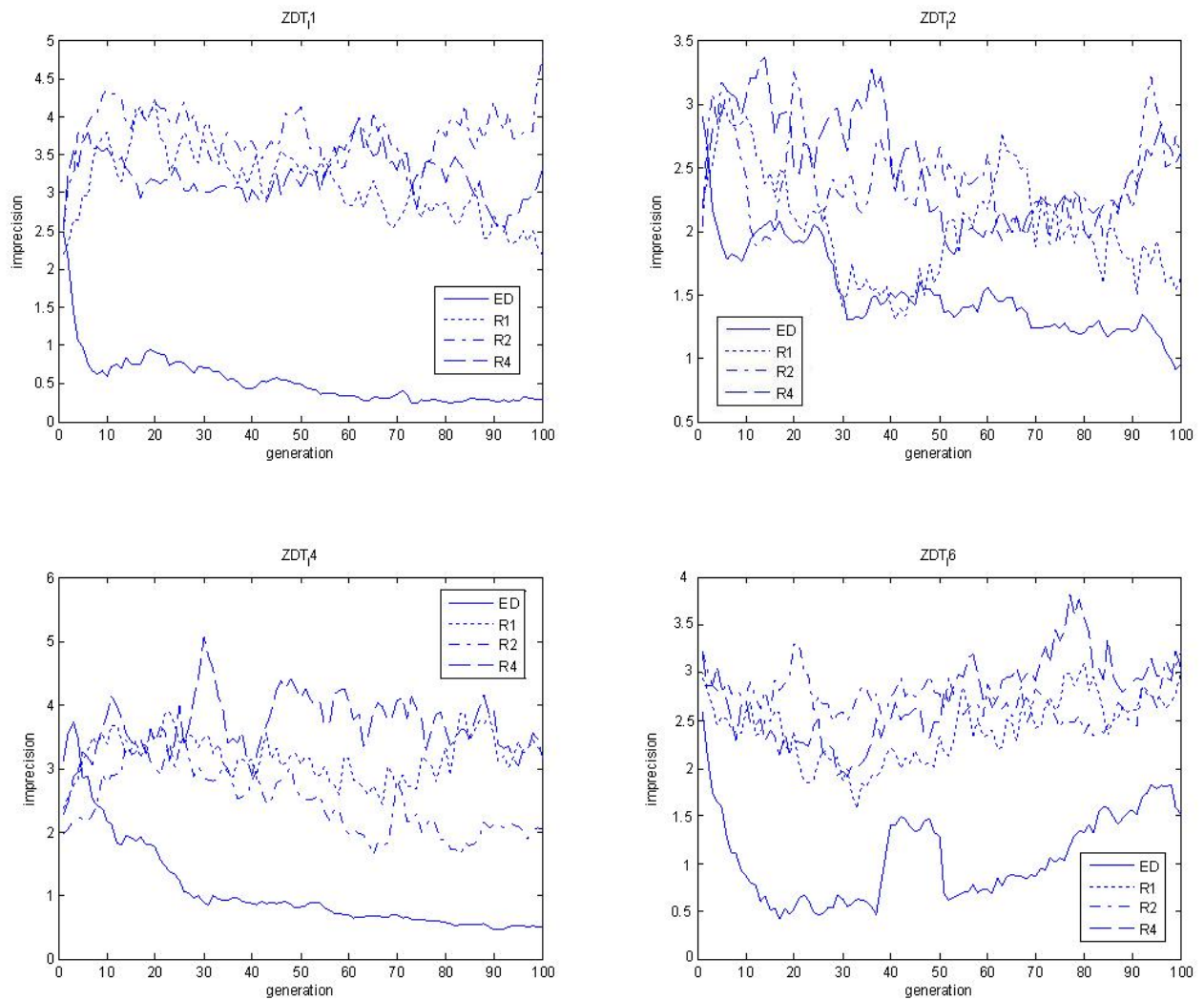

Figure 2: Curves of I metric w.r.t. number of generations 
Table 6: Values of three performance indicators obtained by different interval dominance rules

\begin{tabular}{|c|c|c|c|c|c|c|c|c|c|c|c|c|}
\hline & \multicolumn{2}{|c|}{$\mathrm{ZDT}_{I} 1$} & \multicolumn{3}{|c|}{$\mathrm{ZDT}_{I} 2$} & \multicolumn{3}{|c|}{$\mathrm{ZDT}_{I} 4$} & \multicolumn{4}{|c|}{$\mathrm{ZDT}_{I} 6$} \\
\hline & $\mathrm{H}$ & I & $\mathrm{CPU}$ & $\mathrm{H}$ & I & $\mathrm{CPU}$ & $\mathrm{H}$ & I & $\mathrm{CPU}$ & $\mathrm{H}$ & I & $\mathrm{CP}$ \\
\hline & $\begin{array}{l}\text { Met- } \\
\text { ric }\end{array}$ & $\begin{array}{l}\text { Met- } \\
\text { ric }\end{array}$ & time & $\begin{array}{l}\text { Met- } \\
\text { ric }\end{array}$ & $\begin{array}{l}\text { Met- } \\
\text { ric }\end{array}$ & time & $\begin{array}{l}\text { Met- } \\
\text { ric }\end{array}$ & $\begin{array}{l}\text { Met- } \\
\text { ric }\end{array}$ & time & $\begin{array}{l}\text { Met- } \\
\text { ric }\end{array}$ & $\begin{array}{l}\text { Met- } \\
\text { ric }\end{array}$ & \\
\hline $\mathrm{R} 1$ & $3.70^{*}$ & $2.16^{*}$ & 71 & 3.95 & $1.64^{*}$ & 68 & 4.03 & $3.24^{*}$ & 70 & 3.57 & * & U. \\
\hline $\mathrm{R}$ & $3.66^{*}$ & $4.82^{*}$ & 69 & $3.81^{*}$ & 2.5 & 77 & 4.01 & 2.0 & 72 & 3.77 & $3.24^{*}$ & 75 \\
\hline $\mathrm{R} 4$ & $3.63^{*}$ & $3.32^{*}$ & 71 & 3.84 & $2.61^{*}$ & 79 & $3.95^{*}$ & $3.15^{*}$ & 74 & 3.62 & $3.03^{*}$ & 73 \\
\hline ED & 4.06 & 0.28 & 177 & 4.02 & 0.95 & 187 & 4.07 & 0.50 & 182 & 3.91 & 1.52 & 185 \\
\hline
\end{tabular}

relation at the $5 \%$ significance level, where the statistic result is obtained by a statistic software package SPSS V.19.0.

It can be derived that:

1) the values of $\mathrm{H}$ metric of the final Pareto front generated by the proposed ensemble dominance are significantly superior to those generated by other rules only for a handful number of bi-objective programming problems, indicating that the proposed ensemble dominance relation at least has the similar performance with a single rule on the convergence; nevertheless, users do not bother with which interval dominance rule they should utilize;

2) for four bi-objective programming problems, the value of I metric of the final Pareto front produced by the proposed ensemble dominance is significantly less than those generated by other rules, which implies that the proposed ensemble dominance relation has a competitive performance on imprecision. The likely reason is that the proposed ensemble dominance implicitly involves uncertainty. The advantage of the proposed ensemble framework on imprecision is beyond expectation, and such an advantage will very likely trigger the widespread application of the proposed ensemble framework in interval programming;

3) however, the CPU time of the algorithm embedded by the proposed framework is double longer than those embedded by a single interval dominance rule, which is coincident with Proposition 4.

The above analysis reveals that the reduction strategy is effective to discern the equivalent rules, and the optimal solution(s) produced by the 
proposed framework can better satisfy most of users; the competitive performance of the proposed framework on imprecision and without struggling with which interval dominance rules should be used make it outstanding.

\section{Conclusions}

Interval programming is a widely used technique in real-world situations; however, diverse interval dominance rules hinder its extensive applications.

This paper presents an ensemble framework for integrating interval dominance rules through investigating the correlations of interval dominance rules, developing a strategy to reducing a rule set and assembling the reduced rules. Its advantage is that it can integrate previous interval dominance rules in a framework such that even if users have little knowledge about interval arithmetic, they can use the proposed ensemble dominance to handle their interval programming models without being bothered by the difficulty how to assess interval objective values.

The proposed ensemble framework is tested on three groups of experiments. The empirical results validate that the proposed reduction strategy is effective, and the proposed ensemble dominance relation can generate optimal solutions which satisfy most users; furthermore, it has the excellent performance on reducing imprecision. However, similar to most other ensemble approaches, the method is unavoidably more time-consuming. As stated in Subsection 4.2, there are numerous ways to assemble interval dominance rules, and group decision making can also be borrowed to a great extent. There must exist other economical ensemble ways, which are our future research topics.

\section{Appendix A. The proof of Example 2}

Proof. 1) Given that $\mathbf{x}_{1}$ and $\mathbf{x}_{2}$ are any two solutions of model (2), we prove that $\mathbf{x}_{1} \succ_{R 1} \mathbf{x}_{2} \Longleftrightarrow \mathbf{x}_{1} \succ_{R 3} \mathbf{x}_{2}$. The necessity is firstly to be proved, i.e., $\mathbf{x}_{1} \succ_{R 1} \mathbf{x}_{2} \Longrightarrow \mathbf{x}_{1} \succ_{R 3} \mathbf{x}_{2}$. It can be followed from $\mathbf{x}_{1} \succ_{R 1} \mathbf{x}_{2}$ that $f_{i}^{L}\left(\mathbf{x}_{1}, \mathbf{c}\right) \geq f_{i}^{L}\left(\mathbf{x}_{2}, \mathbf{c}\right), f_{i}^{U}\left(\mathbf{x}_{1}, \mathbf{c}\right) \geq f_{i}^{U}\left(\mathbf{x}_{2}, \mathbf{c}\right)$, and at least one greater-than relation hold, where $i=1,2, \cdots, m$. It can be followed from Eq. (3) that $f\left(\mathbf{x}_{2}, \mathbf{c}\right) \leq_{L U} f\left(\mathbf{x}_{1}, \mathbf{c}\right)$, and there exists at least one $i$, such that $f\left(\mathbf{x}_{2}, \mathbf{c}\right)<_{L U}$ $f\left(\mathbf{x}_{1}, \mathbf{c}\right)$, which implies that $\mathbf{x}_{1} \succ_{R 3} \mathbf{x}_{2}$. The sufficiency can be proved in the same way. 
2) It can be concluded from model (5) that R1 adopts the lower and upper bounds of the objective value to define the relation of solutions. If not-lessthan relation holds for the lower and upper bounds of an interval, the relation also holds for the midpoint of the interval. Nevertheless, it cannot ensure that the relation holds for the width of the interval. That is to say, if solution $\mathbf{x}_{1}$ dominates $\mathbf{x}_{2}$ corresponding to $\mathrm{R} 1, \mathbf{x}_{1}$ and $\mathbf{x}_{2}$ may be incomparable. On the contrary, if the objective value associated with solution $\mathbf{x}_{1}$ contains that associated with solution $\mathbf{x}_{2}$; as long as the midpoint of the objective value associated with solution $\mathbf{x}_{2}$ is not less than that associated with solution $\mathbf{x}_{1}$, $\mathbf{x}_{2}$ dominates $\mathbf{x}_{1}$ corresponding to $\mathrm{R} 2$; however, $\mathbf{x}_{1}$ and $\mathbf{x}_{2}$ are incomparable corresponding to R1. Therefore, R1 and R2 mutually excluded. Similarly, R2 and R4 mutually excluded as well.

3) It can be concluded from model (8) that R4 essentially adopts the midpoint of the objective value to define the relation of solutions. When $\gamma=0.5$, it is necessary that the not-less-than relation holds for the midpoint of an interval. As stated in (2), If not-less-than relation holds for the lower and upper bounds of an interval, the relation also holds for the midpoint of the interval. Therefore, if solution $\mathbf{x}_{1}$ dominates $\mathbf{x}_{2}$ corresponding to $\mathrm{R} 1, \mathbf{x}_{1}$ must dominate $\mathbf{x}_{2}$ corresponding to R4. On the contrary, if the interval of the objective function associated with solution $\mathbf{x}_{1}$ contains that associated with solution $\mathbf{x}_{2}$; as long as the midpoint of the objective value associated with solution $\mathbf{x}_{2}$ is not less than that associated with solution $\mathbf{x}_{1}, \mathbf{x}_{2}$ dominates $\mathbf{x}_{1}$ corresponding to $\mathrm{R} 4$; however, $\mathbf{x}_{1}$ and $\mathbf{x}_{2}$ are incomparable corresponding to R1. Therefore, R1 includes $\mathrm{R} 4$.

Nevertheless, when $\gamma>0.5$, if $\mathbf{x}_{1}$ dominates $\mathbf{x}_{2}$ corresponding to $\mathrm{R} 4$, the midpoint of the objective value associated with solution $\mathbf{x}_{1}$ is greater than that associated with solution $\mathbf{x}_{2}$ in some degree. That is to say, although not-less-than relation holds for the lower and upper bounds of the interval, $\mathbf{x}_{1}$ and $\mathbf{x}_{2}$ may be incomparable corresponding to R4. Combined with the above statement, R1 and R4 mutually excludes under this circumstance.

\section{Appendix B. Descriptions of R5 and R6}

We ever proposed the following interval dominance rule in [13]. For two intervals $a=\left[a^{L}, a^{R}\right]$ and $b=\left[b^{L}, b^{R}\right]$, their maximal interval is denoted as $\beta=\left[\beta^{L}, \beta^{R}\right]$, where the upper and lower bounds of $\beta$ are $\beta^{U}=$ $\max \left\{a^{L}, b^{L}, a^{U}, b^{U}\right\}$ and $\beta^{L}=\max \left\{\left\{a^{L}, b^{L}, a^{U}, b^{U}\right\} \backslash \beta^{U}\right\}$, respectively. A 
possibility degree is defined as follows.

$$
p(a \geq b) \triangleq \frac{d(b, \beta)}{d(a, \beta)+d(b, \beta)}
$$

where $d(a, \beta)$ is the distance between $a$ and $\beta$ whose expression is as follows:

$$
d(a, \beta)=\sqrt{\frac{\left(a^{L}-\beta^{L}\right)^{2}+\left(a^{U}-\beta^{U}\right)^{2}}{2}}
$$

For any objective function $f_{i}\left(\mathbf{x}, \mathbf{c}_{i}\right), i=1,2, \cdots, m$, if the possibility degree of $f_{i}\left(\mathbf{x}_{1}, \mathbf{c}_{i}\right)$ not smaller than $f_{i}\left(\mathbf{x}_{2}, \mathbf{c}_{i}\right)$ is not smaller than that of $f_{i}\left(\mathbf{x}_{2}, \mathbf{c}_{i}\right)$ not smaller than $f_{i}\left(\mathbf{x}_{1}, \mathbf{c}_{i}\right)$, and there exists at least one objective function $f_{q}\left(\mathbf{x}, \mathbf{c}_{q}\right)$, such that the possibility degree of $f_{q}\left(\mathbf{x}_{1}, \mathbf{c}_{q}\right)$ not smaller than $f_{q}\left(\mathbf{x}_{2}, \mathbf{c}_{q}\right)$ is larger than that of $f_{i}\left(\mathbf{x}_{2}, \mathbf{c}_{i}\right)$ not smaller than $f_{i}\left(\mathbf{x}_{1}, \mathbf{c}_{i}\right)$, i.e.,

$$
\mathbf{x}_{1} \succ \mathbf{x}_{2} \Leftrightarrow\left\{\begin{array}{l}
\forall i \in\{1,2, \cdots, m\} \\
p\left(f_{i}\left(\mathbf{x}_{1}, \mathbf{c}_{i}\right) \geq f_{i}\left(\mathbf{x}_{2}, \mathbf{c}_{i}\right)\right) \geq p\left(f_{i}\left(\mathbf{x}_{2}, \mathbf{c}_{i}\right) \geq f_{i}\left(\mathbf{x}_{1}, \mathbf{c}_{i}\right)\right), \\
\exists q \in\{1,2, \cdots, m\}, \\
p\left(f_{q}\left(\mathbf{x}_{1}, \mathbf{c}_{q}\right) \geq f_{q}\left(\mathbf{x}_{2}, \mathbf{c}_{q}\right)\right)>p\left(f_{q}\left(\mathbf{x}_{2}, \mathbf{c}_{q}\right) \geq f_{q}\left(\mathbf{x}_{1}, \mathbf{c}_{q}\right)\right),
\end{array}\right.
$$

$\mathbf{x}_{1}$ is said to dominate $\mathbf{x}_{2}$. This interval dominance rule is denoted as D5.

Zhang et al. defined the following possibility degree [47].

$$
p(a \geq b)=\left\{\begin{array}{l}
1, a^{U} \geq b^{L} \\
\frac{a^{U}-b^{U}}{a^{U}-a^{L}}+\frac{b^{U}-a^{L}}{a^{U}-a^{L}} \times\left(0.5 \frac{b^{U}-a^{L}}{b^{U}-b^{L}}+\frac{a^{L}-b^{L}}{b^{U}-b^{L}}\right), b^{L}<a^{L}<b^{U}<a^{U} \\
\frac{a^{U}-b^{U}}{a^{U}-a^{L}}+0.5 \frac{b^{U}-b^{L}}{a^{U}-a^{L}}, a^{L} \leq b^{L} \leq b^{U} \leq a^{U}
\end{array}\right.
$$

For any objective function $f_{i}\left(\mathbf{x}, \mathbf{c}_{i}\right), i=1,2, \cdots, m, p\left(\mathbf{x}_{1}, \mathbf{x}_{2}\right)=\prod_{i=1}^{m} p_{i}$ $\left(f_{i}\left(\mathbf{x}_{1}, \mathbf{c}_{1}\right) \geq\left(f_{i}\left(\mathbf{x}_{2}, \mathbf{c}_{2}\right)\right)\right.$ is called probability of $\mathbf{x}_{1}$ dominating $\mathbf{x}_{2} ; p\left(\mathbf{x}_{2}, \mathbf{x}_{1}\right)$ $=\prod_{i=1}^{m}\left(1-p_{i}\left(f_{i}\left(\mathbf{x}_{1}, \mathbf{c}_{1}\right) \geq\left(f_{i}\left(\mathbf{x}_{2}, \mathbf{c}_{2}\right)\right)\right)\right.$ is called probability of $\mathbf{x}_{1}$ being dominated by $\mathbf{x}_{2} ; p\left(\mathbf{x}_{1} \| \mathbf{x}_{2}\right)=1-p\left(\mathbf{x}_{1}, \mathbf{x}_{2}\right)-p\left(\mathbf{x}_{2}, \mathbf{x}_{1}\right)$ is called non-dominated probability. If $p\left(\mathbf{x}_{1}, \mathbf{x}_{2}\right)=\max \left\{p\left(\mathbf{x}_{1}, \mathbf{x}_{2}\right), p\left(\mathbf{x}_{2}, \mathbf{x}_{1}\right), p\left(\mathbf{x}_{1} \| \mathbf{x}_{2}\right)\right\}, \mathbf{x}_{1}$ is said to probabilistic dominate $\mathbf{x}_{2}$. This interval dominance rule is denoted as R6. 


\section{Acknowledgment}

This work is jointly supported by National Natural Science Foundation of China with grant No. 61403155, 61375067, 61703188 and 61673404, National Basic Research Program of China (973 Program) with grant No. 2014CB046306-2, and Jiangsu Overseas Research \& Training Program for University Prominent Young \& Middle-aged Teachers and Presidents.

\section{References}

[1] M. Allahdadi, H. M. Nehi, The optimal solution set of the interval linear programming problems, Optimization Letters 7 (8) (2013) 1893-1911.

[2] H. A. Ashayerinasab, H. M. Nehi, M. Allahdadi, Solving the interval linerar programming problem: A new algorithm for a general case, Expert Systems with Applications 93 (2018) 39-49.

[3] L. C. Barbosa, L. F. A. M. Gomes, Assessment of efficiency and sustainability in a chemical industry using goal programming and AHP, Procedia Computer Science 55 (2015) 166-174.

[4] A. K. Bhunia, S. S. Samanta, A study of interval metric and its application in multi-objective optimization with interval objectives, Computers\&Industrial Engineering 74 (2014) 169-178.

[5] G. R. Bitran, Linear multiobjective problems with interval coefficients, Management Science 26 (1981) 694-706.

[6] S. Chanas, D. Kuchta, Multiobjective programming in optimization of the interval objective functions-a generalized approach, European Journal of Operational Research 94 (3) (1996) 594-598.

[7] K. Deb, A. Pratap, S. Agarwal, T. Mrystivan, A fast and elitist multiobjective genetic algorithm: NSGA-II, IEEE Transactions on Evolutionary Computation 6 (2) (2002) 182-197.

[8] R. L. Dong, C. Zong, M. Q. Li, An interactive genetic algorithm with the interval arithmetic based on hesitation and its application to achieve customer collaborative product configuration design, Applied Soft Computing 38 (1) (2016) 384-394. 
[9] Y. R. Fan, G. H. Huang, A robust two-step method for solving interval linear programming problems within an environmental management context, Journal of Environmental Informatics 19 (1) (2012) 1-9.

[10] Y. R. Fan, G. H. Huang, Y. P. Li, Robust interval linear programming for environmental decision making under uncertainty, Engineering Optimization 44 (11) (2012) 1321-1336.

[11] F. J. Gao, W. Z. Yang, Possibility degree and its application to multiple attribute interval decision making, Mathematics in Practice and Theory 44 (22) (2014) 161-166

[12] C. K. Goh, K. C. Tan, Handling noise in evolutionary multi-objective optimization, Studies in Computational Intelligence 186 (2009) 55-99.

[13] D. W. Gong, N. N. Qin, X. Y. Sun, Evolutionary optimization algorith$\mathrm{m}$ for multi-objective optimization problems with interval parameters, in: Proceedings of 5th IEEE International Conference on Bio-Inspired Computing: Theories and Applications, 2010, pp. 411-420.

[14] D. W. Gong, N. N. Qin, X. Y. Sun, Evolutionary algorithms for optimization problems with uncertainties and hybrid indices, Information Sciences 181 (19) (2011) 4124-4138.

[15] D. W. Gong, J. Sun, Z. Miao, A Set-based genetic algorithm for interval many-objective optimization problems, IEEE Transactions on Evolutionary Computation, DOI 10.1109/TEVC.2016.2634625.

[16] Y. Han, Y. F. Huang, G. Q. Wang, I. Maqsood, A multi-objective linear programming model with interval parameters for water resources allocation in dalian city, Water Resource Management 25 (2011) 449-463.

[17] M. Hladík, Complexity of necessary efficiency in interval linear programming and multiobjective linear programming, Optimization Letters 6 (5) (2012) 893-899.

[18] M. Hladík, How to determine basis stability in interval linear programming, Optimization Letters 8(1) (2014) 375-389.

[19] M. Hladík, On strong optimality of interval linear programming, Optimization Letters (2016) 1-10. 
[20] M. Inuiguchi, M. Sakawa, Minimaxregreat solution to linear programming problems with an interval objective function, European Journal of Operational Research 86 (3) (1995) 526-536.

[21] H. Ishibuchi, H. Tanaka, Multiobjective programming in optimization of the interval function, European Journal of Operational Research 48 (2) (1990) 594-598.

[22] C. Jiang, X. Han, F. J. Guan, Y. H. Li, An uncertain structural optimization method based on nonlinear interval number programming and interval analysis method, Engineering Structures 29 (11) (2007) 31683177 .

[23] C. Jiang, Z. G. Zhang, Q. F. Zhang, X. Han, H. C. Xie, J. Liu, A new nonlinear interval programming method for uncertain problems with dependent interval variables, European Journal of Operational Research 238 (1) (2014) 245-253.

[24] H. Karshenas, C. Bielza, P. Larrañaga, Interval-based ranking in noisy evolutionary multi-objective optimization, Computational Optimization and Applications 61 (2015) 517-555.

[25] V. Kreinovich, Interval uncertainty as the basis for a general description of uncertainty: A position paper, International Journal of Intelligent Technologies and Applied Statistics, to be appeared.

[26] W. Li, J. J. Luo, Q. Wang, Y. Li, Checking weak optimality of the solution to linear programming with interval right-hand side, Optimization Letters 8 (4) (2014) 1287-1299.

[27] P. Limbourg, D. E. S. Aponte, An optimization algorithm for imprecise multi-objective problem function, in: Proceedings of IEEE Congress on Evolutionary Computation, 2005, pp.459-466.

[28] Q. G. Lin, Q. Wu, G. H. Huang, M. Y. Zhai, An interval parameter optimization model for sustainable power systems planning under uncertainty, Electrical Power and Energy Systems 54 (2014) 631-641.

[29] S. T. Liu, Using geometric programming to profit maximization with interval coefficients and quantity discount, Applied Mathematics and Computation 209 (2) (2009) 259-265. 
[30] J. J. Luo, W. Li, Strong optimal solutions of interval linear programming, Linear Algebra and its Applications 439(8) (2013) 2479-2493.

[31] N. Marković, R. Nair, P. Schonfeld, E. Miller-Hooks, M. Mohebbi, Optimizing dial-a-ride services in Maryland: Benefits of computerized routing and scheduling, Transportation Research Part C: Emerging Technologies 55 (2015) 156-165.

[32] W. L. Oberkampf, J. C. Helton, C. A. Joslyn, S. F. Wojtkiewicz, S. Ferson, Challenge problems: Uncertainty in system response given uncertain parameters, Reliability Engineering and System Safety 85 (1-3) (2004) 11-19.

[33] C. Oliveira, C. H. Antunes, Multiple objective linear programming models with interval coefficients- An illustrated overview, European Journal of Operational Research 181 (3) (2007) 1434-1463.

[34] R. Osuna-Gómez, B. Hernández-Jiménez, Y. Chalco-Cano, G. RuizGarzón, New Efficiency conditions for multiobjective interval-valued programming problems, Information Sciences 420 (2017) 235-248.

[35] Q. Pang, H. Wang, Z. S. Xu, Probabilistic linguistic term sets in multiattribute group decision making, Information Sciences 369 (10) (2016) 128-143.

[36] J. R. Prague, Strong solvability of interval linear programming problems, Computing 26 (1) (1981) 79-82.

[37] S. Rivaz, M. A. Yaghoobi, Minimax regret solution to multiobjective linear programming problems with interval objective functions coefficients, Central European Journal of Operations Research 21 (3) (2013) 625-649.

[38] L. Sahoo, A. K. Bhunia, and P. K. Kapur, Genetic algorithm based multi-objective reliability optimization in interval environment, Computers \& Industrial Engineering 62 (1) (2012) 152-160.

[39] J. Sun, D. W. Gong, Solving interval multi-objective optimization problems using evolutionary algorithms with lower limit of possibility degree, Chinese Journal of Electronics 22 (2) (2013) 269-272. 
[40] J. Sun, D. W. Gong, Z. Miao, Z. G. Xu, H. Zhang, Ensemble dominance for solving interval programming problems, in: Proceedings of IEEE Congress on Evolutionary Computation, 2016, pp.562-569.

[41] A. Violin, Mathematical programming approaches to pricing problems, PhD thesis (2014), Belgium: Université Libre de Bruxelles.

[42] Z. S. Xu, Q. L. Da, Research on method for ranking interval numbers, Systems Engineering 19 (6) (2001) 94-96.

[43] H. Wang, Extended hesitant fuzzy linguistic term sets and their aggregation in group decision making, International Journal of Computational Intelligence Systems 8 (1) (2015) 14-33.

[44] H. Wang, Z. S. Xu, Some consistency measures of extended hesitant fuzzy linguistic preference relations, Information Science 297 (3) (2015) 316-331.

[45] X. Q. Wang, G. H. Huang, Violation analysis on two-step method for interval linear programming, Information Sciences 281 (2014) 85-96.

[46] K. Zaman, S. Rangavajhala, M. P. McDonald, S. Mahadevan, A probabilistic approach for representation of interval uncertainty, Reliability Engineering and System Safety 96 (1) (2011) 117-130.

[47] Y. Zhang, D. W. Gong, G. S. Hao, Y. Q. Jiang, Particle swarm optimization for multi-objective systems with interval parameters, Acta $\mathrm{Au}$ tomatica Sinca 34 (8) (2008) 921-928.

[48] Z. H. Zhang, X. X. Wang, J. X. Lu, Multi-objective immune genetic algorithm solving nonlinear interval-valued programming, Engineering Applications of Artificial Intelligence 67 (2018) 235-245.

[49] Z. H. Zhao, X. Han, C. Jiang, X. X. Zhou, A nonlinear interval-based optimization method with local-densifying approximation technique, Structure Multidisplinary Optimization 42 (4) (2010) 559-573. 